\title{
Financial Benefit Analysis of an Electric Water Heater with Direct Load Control in Demand Response
}

\author{
Md Tofael Ahmed, Pedro Faria, Zita Vale \\ GECAD - Research Group on Intelligent Engineering and Computing for Advanced Innovation and Development \\ Polytechnic of Porto \\ Porto, Portugal
}

mdtof@isep.ipp.pt, pnf@isep.ipp.pt, zav@isep.ipp.pt

\begin{abstract}
The peak demand reduction during peak hour is a challenge to the retail energy providers. Demand response program plays a major role to fulfil this purpose. The small household appliances like electric water heater can participate in the demand response program by aggregating it in the smart building energy management system. This paper discusses demand response possibilities of a residential electric water heater, the overall consumption profile, temperature profile and the financial benefit in the consumer level. The direct load control demand response method in yearly timeframe is proposed and applied. Realtime electricity pricing with incentive-based demand response is considered and applied to the direct load control with financial benefit to the consumers. The study includes the difference between normal consumption and consumption after using DLC, normal temperature profile and temperature profiling after DLC. The results exhibit that there is significant energy consumption reduction in the consumer level without making any discomfort.
\end{abstract}

Keywords-Building Energy Management System, Demand Response, Direct Load Control, Financial Benefit.

\section{INTRODUCTION}

Electricity is one of the most important factors for the technological and social development. It is facing fundamental changes due to development of smart energy technology infrastructure in recent years [1]. There is a fast deployment of this technology with distributed energy resources and several renewable energy technologies. This fast growth is making complexity to maintain a reliable and economic daily operation of traditional power grids. These renewable technologies and distributed resources will also add uncertainty to the local grid [2]. The introduction of smart grid technology is based on the information and communication assistance for supporting renewables and distributed generations. The power suppliers as well as end users are also benefitted by the use and development of this technology [3].

Demand response (DR) helps to maintain balance between load and power generation by encouraging customers to change or reduce their consumption schedule [4]. The traditional use of demand response is on the bulk power to fulfil its operational and planning activities [5]. Actually, DR helps to modify the users' normal electricity consumption patterns with electricity price schedules patterns or incentive payments to keep lower electricity usage. A price-responsive DR management system is introduced in [6]. DR method is implemented traditionally in the industries and other large factories. Now, it is also necessary to implement DR events in small residential houses. Because the total energy consumption in the buildings is $35-40 \%$ with $50-65 \%$ electricity consumption in the developed countries [7]. The consumption in the buildings of developing countries will also increase due to industrialization and development of technological infrastructure [8]. All used smart appliances and load can be considered for establishing DR events. After analysing all these factors, it can be considered that the small house appliances are able to participate in DR programs. The electrical household appliances are considered as the major parts of the house due to using necessity [9]. Due to the rapid increase of load density in residential sectors the power consumption will increase too. This is considered as one of the major ongoing problem for traditional electrical grid infrastructure [10].

The domestic electric water heater (EWH) can have a huge potential for the implementation of residential DR events. The enormous flexibility of EWHs and its usage make it an appropriate candidate to participate in the demand response programs. Electric water heater consumes a major power $(7.5 \%$ to $40 \%$ ) of the residences total energy consumption [6]. The switching actions in that type of heaters is very flexible as the elements are resistors and there is no support of reactive power needed from the grid. There is also no energy waste for balancing services and these heaters can be used as energy storage additionally [11]. The load shifting method and shaving services make it very useful for the home energy management system.

Load modelling is also necessary for the customers to estimate the overall power consumption. To receive signals from the utility including the smart grid technology expansion and implementation of DR control strategy in residential buildings it is also required [12]. It is not easy rather challenging to understand load models though several studies and uses regarding load model has already done in last years. To obtain precise load models with new techniques a brief study is necessary which can match simulated behaviours with measured real data [13]. It is significant to study the residential DR events at the distribution circuit level also to study consumer action. DR implementation in residential household appliances increase the customers power consumption reduction activities very efficiently. It can also maintain the high fluctuations of power supply with higher renewable energy generations penetration that can make a possible stable solution for the power system. Prior agreements for DR signals are established between the end users and the utilities to consider the pricing method $[14,15]$. Building Energy Management System can make a link between the consumers and the electricity providers for doing so. It is capable of provisioning DR and efficient control of local energy inside the territory. It consists of home energy management system (HEMS) which can study the behaviours of consumers that is achieved by modelling of home appliance [16].

In this paper, improved modelling of electric water heater $(\mathrm{EWH})$ has been developed based on the physical characteristics of the appliances. A case analysis with real data of an EWH has been studied to validate its participation in DR event with the integration of HEMS [17]. It is the key factor to the electricity operational cost reduction, peak load reduction that can minimize the energy waste and creates eco-friendly management. It can take participation in the DR without any human interaction [18]. During recent years, the expansions of 
HEMS with advanced technologies and smart metering system, smart monitoring of household consumptions are occurred rapidly [19].

As we want to analyse the potentiality of DR participation of our EWH, we should consider the load modelling. It can establish a connection between the overall consumption and its parameters. Water temperature, ambient temperature, water usage and physical characteristics are considered as the EWH parameters. For this work, a single element electric water heater for residential household is considered. This EWH has a beneficial point that is it can classify the thermodynamic model by using heater power measurements only. Also, it is easy to clarify and can simulate different loads very efficiently. A thermodynamic model for a single EWH can be describe by [20]:

$$
\begin{aligned}
& \mathrm{Tt}^{1}{ }^{1} \mathrm{Qt}-\mathrm{GTt}-\mathrm{T}_{\text {out }}-\mathrm{c} \mathrm{W} \\
& \text { C } \\
& \text { P0 } \\
& \text { Tt Tlow } \\
& \text { P t } 0 \quad \text { Tt } T_{\text {high }}
\end{aligned}
$$$$
\text { D t T t - Tin }
$$

Qt Pt ut

where $\mathrm{C}$ is thermal capacity, $\mathrm{Qt}$ is heater power, $\mathrm{G}$ is thermal resistance, $T_{\text {out }}$ is the ambient temperature, is water density, $c_{p}$ is specific heat, $\mathrm{W}_{\mathrm{D}}$ is water demand, $\mathrm{T}_{\text {in }}$ is inlet water temperature.

For the controlling of EWH while it participates in DR a set of conditions are required for the observation purpose and to know the device's operation status. The device status indicates whether it can be operated for DR participation or it cannot be operated. The device status mathematical expression can be stated as [18]:

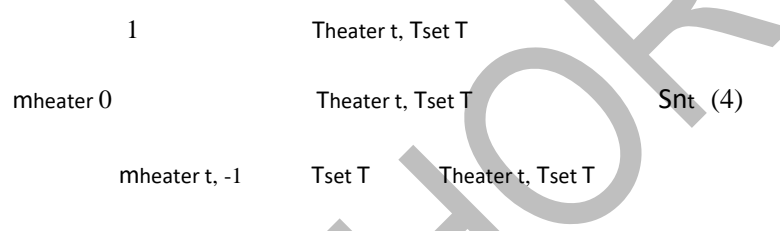

Here, $\mathrm{T}$ is the dead band temperature which is $\pm 2^{\circ} \mathrm{C}$ and $\mathrm{Sn}_{\mathrm{t}}$ is the DR signal. The DR signal controls the electric power demand of the water heater. The signal originated from the revised thermostat set point can be modified by the consumer at the time of DR events.

The rest of paper is organized as follows. Section II represents the description regarding EWH usage profile. The proposed method for the heater's participation in the demand response program is represented in Section III. A DR case study in Section IV is briefly discussed. The performance of proposed system, and its results with $f$ are provided in Section V. Finally, the main conclusions of the paper are presented in Section VI.

\section{EWH USAGE PROFILE}

It is already mentioned that a single element electrical water heater's consumption profile is considered for the analysis purpose. The heater one-year data is taken from our research group (GECAD) office building. It is the only existing water heater in our building which is used by several people. The temperature sensor is placed just outside the heater is able to measure the outlet water temperature in real time. To maintain a comfort temperature level for the user a set temperature is also considered to compare the changes also. Fig. 1 represents the daily overall average consumption profile of the heater for a year period. To get more clear idea about the consumption profile Fig. 2 is represented which shows the monthly average consumption data for the EWH. It also describes that there are few selected months have higher consumption period.

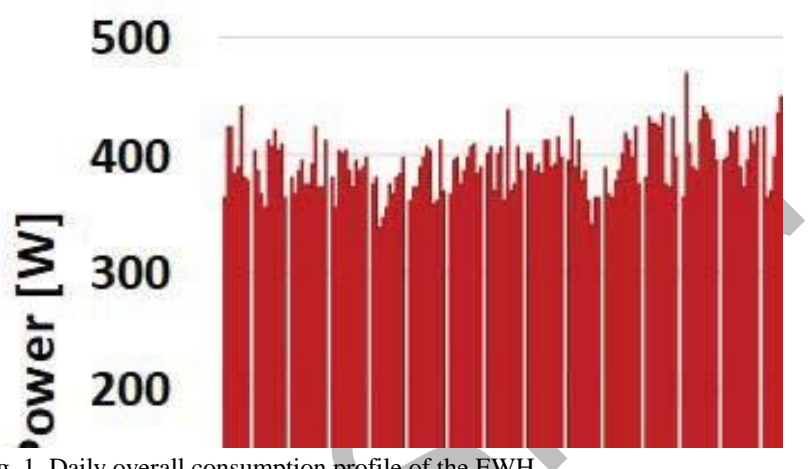

Fig. 1. Daily overall consumption profile of the EWH.

13,50

\section{3,00}

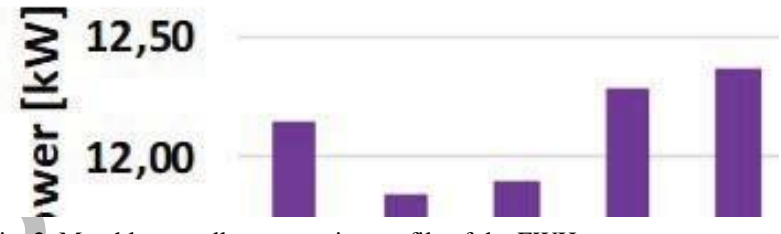

Fig. 2. Monthly overall consumption profile of the EWH.

Maintaining temperature comfort level for the users during the DR participation of the EWH is a challenge. Fig. 3 represents the average temperature profile of the EWH for a

year. From the illustrated figure it appears that the consumers temperature comfort level is around $40^{\circ} \mathrm{C}$. There is also a set temperature level for the investigated water heater so that it can follow the consumers required temperature comfort level. Fig. 4 and Fig. 5 represents the daily and monthly average electricity cost for the heater. Initial market real time pricing data are taken form Portuguese sector of Iberian Electricity Market (MIBEL) (www.omie.es). Those figures abbreviate the daily and monthly pricing schedule with the total cost during the year.

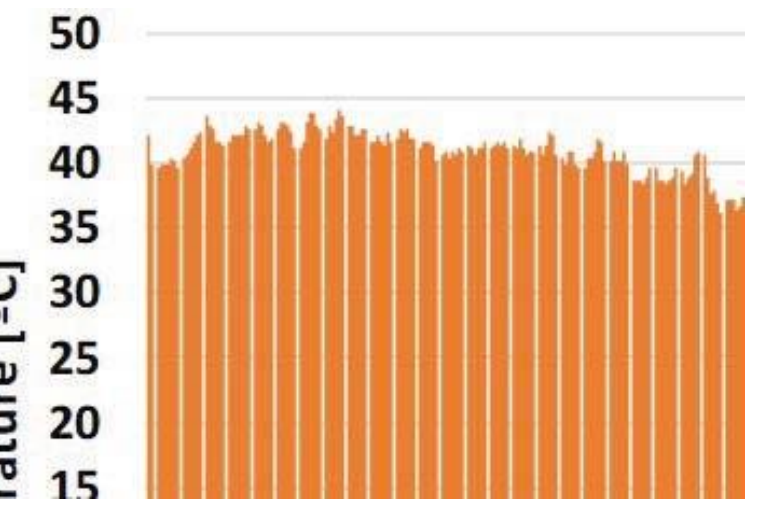

Fig. 3. Daily overall temperature profile of the EWH.

From the Fig. 4 and Fig. 5 it is clear that the electricity cost is higher in the winter season like in October, November and December. It is also observed that the temperature does not fluctuate to a significant level during its activation period. The temperature profile is an 
important factor to be considered and observed during the DR participation for our proposed appliance.

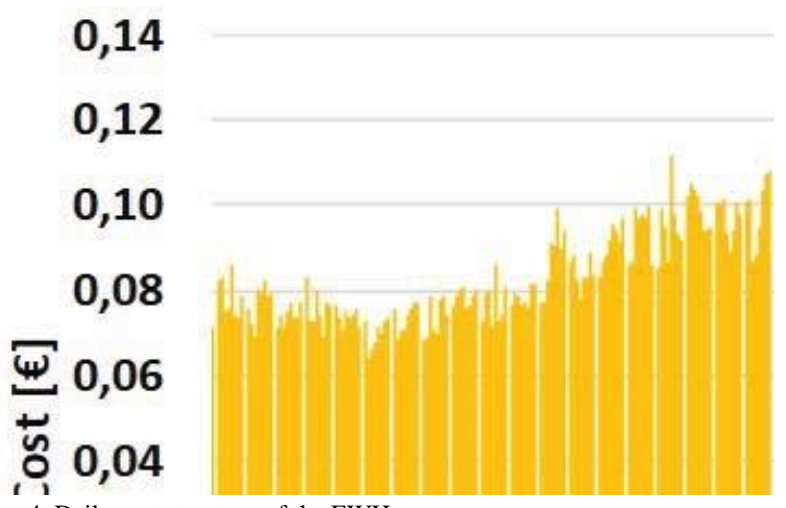

Fig. 4. Daily cost structure of the EWH.

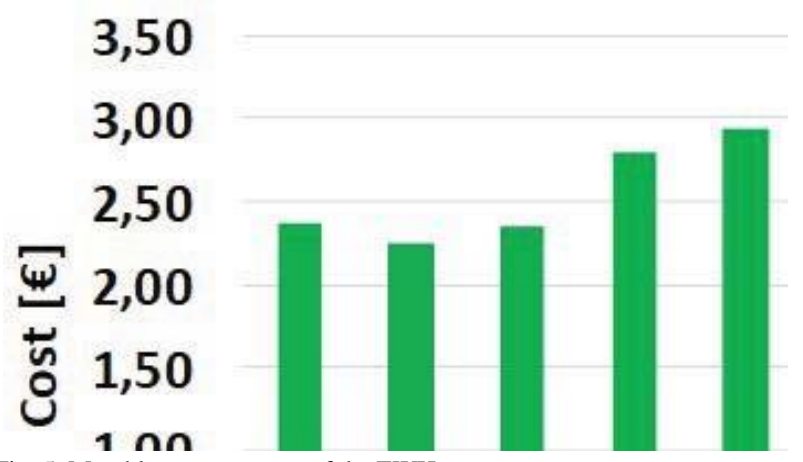

Fig. 5. Monthly cost structure of the EWH.

\section{PROPOSED ANALYSIS}

There are two types of DR method existing in the current electricity market are price-based DR and incentive-based DR [21]. In Price-based DR method, the consumers change the consumption pattern to change in purchase price. It has different kinds are Real time pricing (RTP), time-of-use (TOU) and critical-peak pricing (CPP). In incentive-based DR customers receive fixed or time varying incentives to be added to their electricity rates. It is a program that motivates customer to change their consumption pattern in order to reduce peak load consumption and lower the electricity consumption to avoid imbalances in the electricity grid.

Among several types of incentive-based DR programs, Direct Load Control (DLC) is considered for this work. In this controlling method, the aggregator control (shut down or change of cycles) the electrical equipment remotely [22]. A control event for DLC is briefly discussed in [23].

Before adapting the DR DLC method in the proposed heater, we classified the consumption data into three periods after analysing the consumption profile available in Fig. 2. The high usage months (HUM), medium usage months (MUM) and low usage months (LUM) are classified based on consumption profile.

The considered months for HUM are December, January, March and May. The months in MUM are July, October, November, April and June. The months in LUM are August, September and February. In HUM, DLC is offered for any six days from every month considering any five hours control by the aggregator. In MUM, DLC is offered for any five days from every month considering any four hours control by the aggregator. In LUM, DLC is offered for any two days from every month considering any five hours control by the aggregator. The proposed method of the DLC agreement with numerical values from consumer side is shown in the table I.

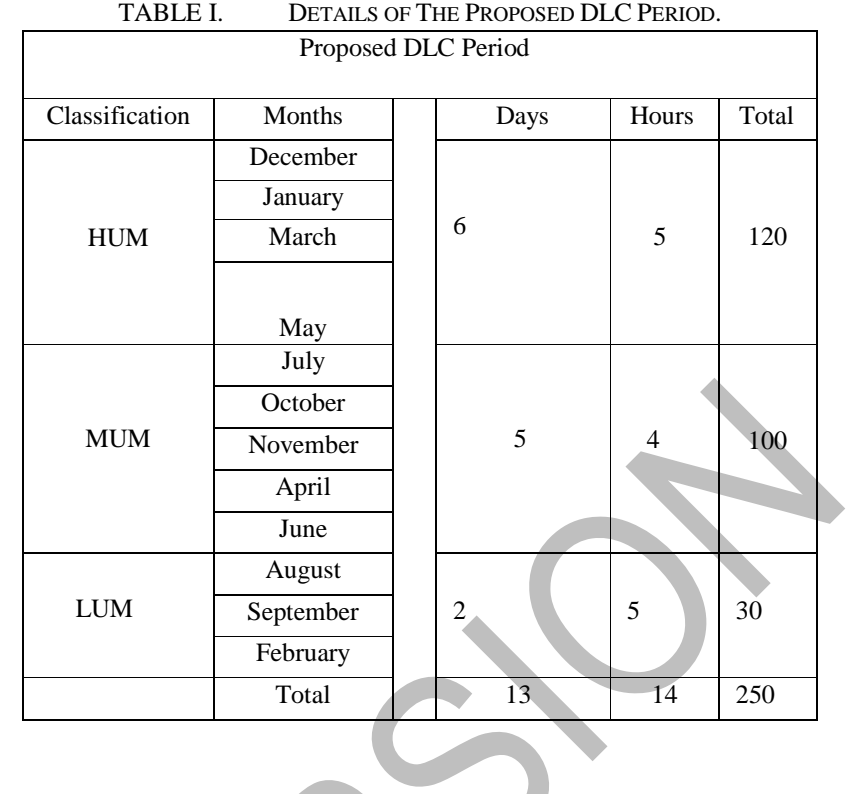

Direct load control (DLC) method offers incentive to the customers additionally too. In incentive-based DR method it is necessary to have an agreement between the consumer and the retail energy provider. The details of the incentive plan from provider is shown in the table II.

TABLE II. DLC INCENTIVE PLAN.
\begin{tabular}{|c|c|c|}
\hline \multicolumn{3}{|c|}{ Proposed Incentive Plan } \\
\hline No. of Hours & Rate $(€ /$ hour $)$ & Total Incentive \\
\hline 250 & 0,05 & 12,5 \\
\hline
\end{tabular}

The table II describes the proposed incentive plans from the energy provider or aggregator side for the EWH DR program. It is an hourly incentive-based financial program. The total hour is 250 and the hourly rate is $0,05 €$. The total obtainable incentive for a year is $12,5 €$.

\section{DR CASE STUDY}

This section describes the case study of the work which considers the possibility of a single element electric water heater's participation in DR events. The study is based on the EWH's average daily electricity consumption, temperature profile, output behaviour and consumers comfort. This profiling is divided into three main parts based on the consumption months (HUM, MUM, and LUM).

Fig. 6 illustrates the difference between average daily consumption profile and average consumption profile after proposing DLC for HUM period. It indicates that the consumption in HUM period is very high. But, the consumption reduces to a significant level with the use of proposed demand response DLC method over the end-user consumption. 
0,60

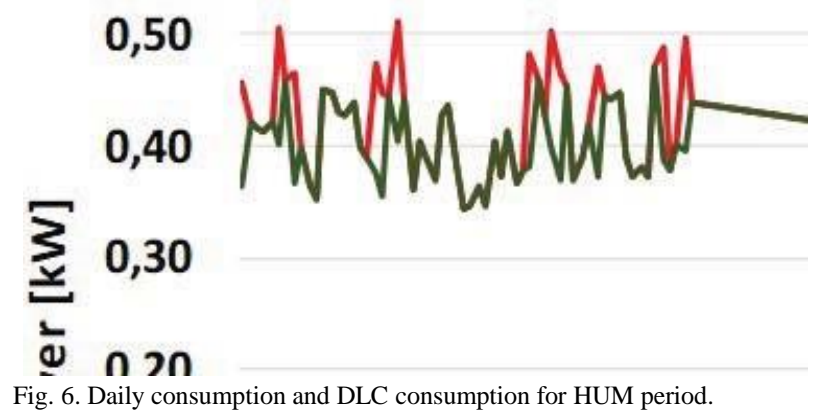

Fig. 7 illustrates the difference between daily average consumption profile and consumption profile after DLC of the EWH for MUM period. In this case study it can be seen from the figure that the consumption is medium during the MUM months. Also, it appears that the consumption profile has significant reduction after the execution of proposed DR program. Fig. 8 illustrates the difference between average daily consumption profile and consumption profile after DLC of the EWH for LUM period.

\section{0,60}

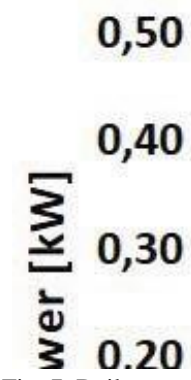

The details of the heater's load reduction profiles are shown in the table III below. The table provides average overall monthly reduced consumption with the reduced percentages. The results indicate that the three consumption periods of the appliance have three different consumption level based on the category. In the results it can be seen that the higher usage months has higher load reduction with higher percentage. The average usage months has medium load consumption reduction as the consumers has medium consumption during those months. The overall percentage is also in medium level. Lastly, the lower usage months have the lowest consumption in the year with lowest number of months.

From the provided obtained output result it is suggested that the DLC DR program has positive attitude towards the residential electrical appliances like EWH. The peak load reduction during higher consumption is also magnificent. During the operation the heater is turned off for a certain period which saves a certain amount of electricity consumption and can help to balance the imbalances occur by the renewable energy generations and other distributed energy resources.

TABLE III. TOTAL LOAD REDUCTION BY DLC METHOD.

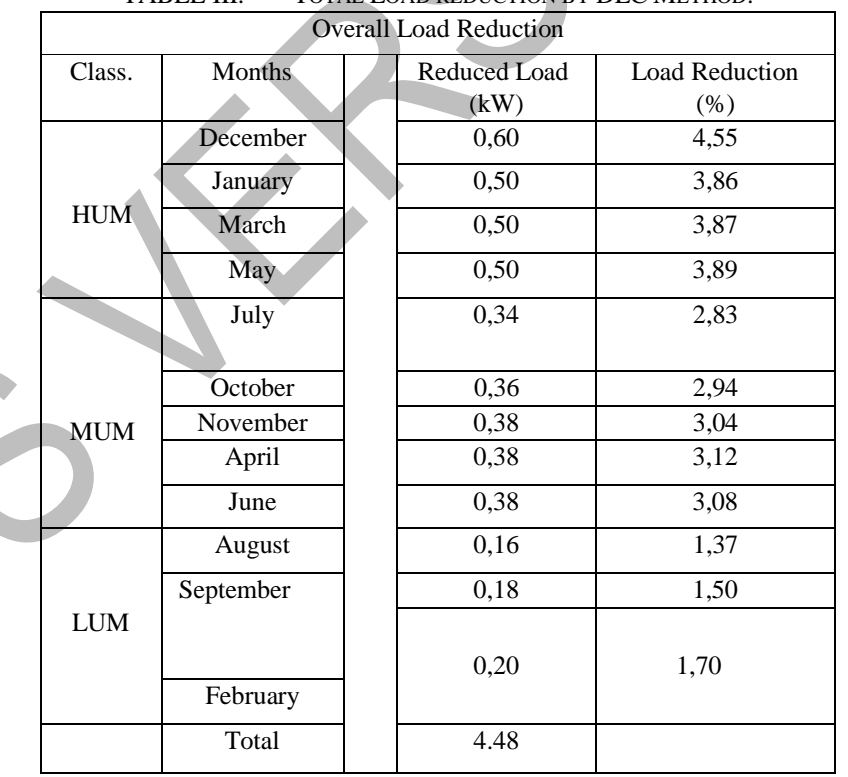

The proposed method offers incentives which is financial gain with load reduction is also considered as another benefit. Fig. 9 describes the financial profit involvement in the proposed DR method for HUM period. It shows the overall profit obtained by the consumers for allowing this operation for implementation in the heater.

\section{RESULTS}

The case study is solved by using DLC method [21] for an electric water heater by using an incentive-based [16] demand response program. To reduce peak load during peak consumption DLC is a widely recognized DR method. The results from the analysis show a significant amount of electricity usage reduction using proposed method. 
6,00

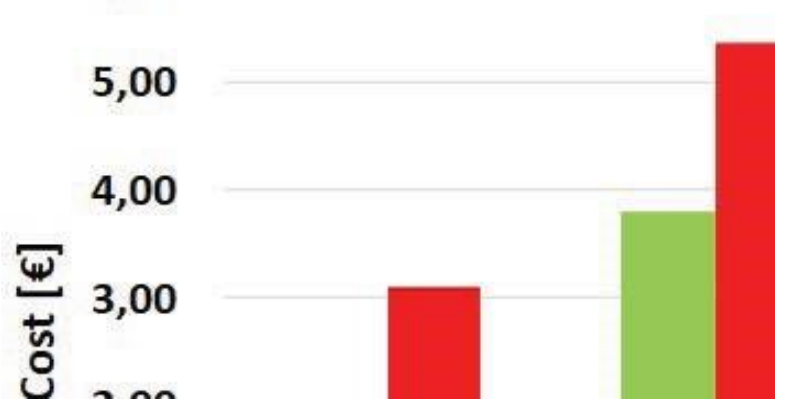

Fig. 9. Overall cost saved and overall monthly cost for all the HUM period.

Here, it describes the total money saved by the reduced load consumption and the incentive money they receive from the aggregator for the DR participation. The cost save part mentioned in the figure describes monthly cost reduced by reduced consumption and the monthly cost part explains the normal average cost for a month. It also shows that December and January months have higher consumption, so these months have more possibility for participating in DR programs that can provide financial benefit.

Fig. 10 illustrates the difference between the overall the monthly cost saved by our proposed load control method. The considered months are the average usage months of the year. The monthly total saved cost is calculated by adding the obtained cost by reducing the peak load consumption and the total incentives provided by the aggregator. It shows a significant benefit in the MUM period too. It can be a

profitable achievement for the users of a small residential houses. The months of October and November have higher consumption so as the greater benefit.

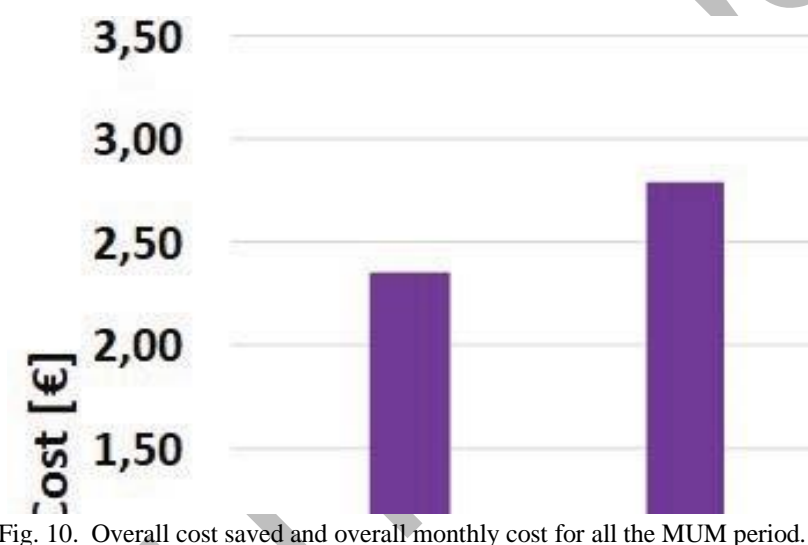

Fig. 10. Overall cost saved and overall monthly cost for all the MUM period.

Similarly, Fig. 11 presents the overall cost for the heater and the cost saved by the proposed DLC DR method in the low usage months. Though the number of months in LUM period is the least in the year but the financial profit can also gain from this period. The figure depicted here tells that February month has the highest consumption in that period. The cost saved part explains the overall cost can be saved by using the controlling method and monthly cost describes the overall average cost per month here.

Fig. 11. Overall cost saved and overall monthly cost for all the LUM period.

The considered EWH is appropriate to participate in the proposed DLC demand response method. It not only reduced the consumption of the consumers but also contributes in the consumer's financial area. The temperature data analysed from there shows that the
3,00

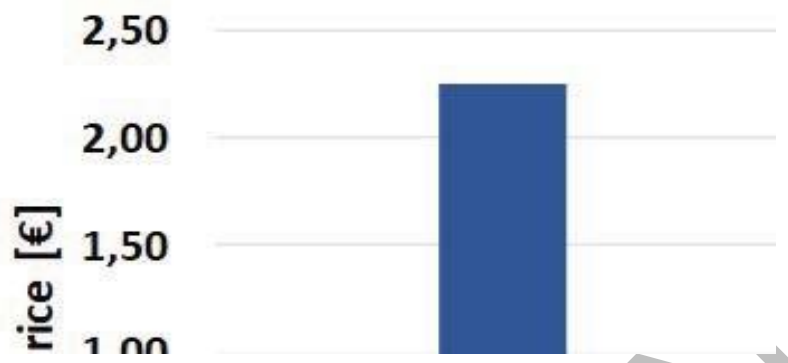

temperature reduction in the water during the operation is not so significant that can hamper the comfort level.

\section{CONCLUSIONS}

The estimation of the load reduction impact here signifies that direct load control to the small residential electrical appliances can be an effective tool for the peak load reduction during peak demand. Also, this method can achieve financial benefit to the consumers. This paper reviews an optimizationbased home energy management system for small residential electrical appliances. The electrical water heater is considered to justify the possibility of demand response participation. The physical and operational characteristics of an EWH is also discussed here.

By developing proposed model and analysing the characteristics of the EWH it is possible to develop an aggregated direct load control system for the device. The experimental real data of the electric water heater is considered for the analysis purpose and direct load control method is proposed to control the heater. The classification of the months based on the consumption is also discussed here. The restricted consumption for the heater by using DLC and its influence on the consumers behaviour is also shown here. Though DLC method is an old technique it can maximize load reduction for a specific control period. The main problem for DLC is during interval only one control strategy can be applied to each of the device.

The duration of load controlling by the aggregator, considered length of this duration and the reduced power consumption is also discussed. The incentives offer for the consumers from the aggregator side is explained and the output result is also studied in this paper. After analysing the whole picture of the implemented method, it is concluded that the participation of the EWH to the proposed DR is beneficial for both the aggregator and consumer. The load consumption reduction and financial benefit is so significant that the possibility of this residential EWH to participate in the demand response DLC program is more conventional. A single element water heater is discussed here as it is the only existing heater of our building. This method can be implemented for dual element water heater or any other heater analysis purpose. This work has a few shortcomings which will be improved in future work.

\section{ACKNOWLEDGEMENTS}

The present work was done and funded in the scope of the following projects: SIMOCE (ANI|P2020 17690); and UID/EEA/00760/2013 funded by FEDER Funds through COMPETE program and by National Funds through FCT.

\section{REFERENCES}

[1] Uros Markovic, "Fast Demand Response with Cooling Devices," Semester Thesis, PSL 1411, Swiss Federal Institute of Technology (ETH) Zurich, June $15,2014$. 
[2] Ruisheng Diao, Zhang, and N. Samaan, "Electric water heater modeling and control strategies for demand response," in 2012 IEEE Power and Energy Society General Meeting, 2012, pp. 1-8.

[3] M. S. Ahmed, A. Mohamed, R. Z. Homod, H. Shareef, and K. Khalid, International journal of applied engineering research., vol. 11, no. 16. Research India Publications, 2006.

[4] L. Zeng, Y. Sun, Q. Ye, B. Qi, and B. Li, "A centralized demand response control strategy for domestic electric water heater group based on appliance cloud platform," IEEJ Trans. Electr. Electron. Eng., vol. 12, pp. S16-S22, Dec. 2017.

[5] Federal Energy Regulatory Commission, "Assessment of Demand Response and Advanced Metering," December 2017, United States.

[6] B. Lin, S. Li, and Y. Xiao, "Optimal and Learning-Based Demand Response Mechanism for Electric Water Heater System," Energies, vol. 10, no. 11, p. 1722, Oct. 2017.

[7] M. A. Riffat SB, "Building Energy Consumption and Carbon dioxide Emissions: Threat to Climate Change," J. Earth Sci. Clim. Change, vol. 3, Jan. 2015

[8] N. Lior, "Energy resources and use: The present situation and possible paths to the future," Energy, vol. 33, no. 6, pp. 842-857, Jun. 2008 .

[9] F. Fernandes, H. Morais, Z. Vale, C. Ramos, 專 Dynamic load management in a smart home to participate in demand response events,尉 Energy Build., 82, pp. 592-606, October 2014.

[10] P. Faria, J. Spínola and Z. Vale, 專Reschedule of Distributed Energy Resources by an Aggregator for Market Participation,尉 Energies, vol. 11(16), pp. 713, March 2018.

[11] Z. Xu, R. Diao, S. Lu, J. Lian, and Y. Zhang, 專Modeling of Electric Water Heaters for Demand Response: A Baseline PDE Model, 尉 IEEE Transactions on Smart Grid, vol. 5(5), pp. 2203-2210 September 2014

[12] R. Z. Homod, K. S. M. Sahari, H. A. Almurib, and F. H. Nagi, "Double cooling coil model for non-linear HVAC system using RLF method," Energy and buildings, vol. 43(9), pp. 2043-2054, September 2011.

[13] P. Siano, "Demand response and smart grids將A survey," Renewable and Sustainable Energy Reviews, vol. 30, pp. 461-478, February 2014.

[14] H. T. Haider, O. H. See, and W. Elmenreich, "A review of residential demand response of smart grid," Renewable and Sustainable Energy Reviews, vol. 59, pp. 166-178, June 2016.

[15] M. Beaudin and H. Zareipour, "Home energy management systems: a review of modelling and complexity," Renewable and Sustainable Energy Reviews, vol. 45, pp. 318-335, May 2015.

[16] S. Rotger-Griful, U. Welling, and R. H. Jacobsen, "Implementation of a building energy management system for residential demand response," Microprocess. Microsyst., vol. 55, pp. 100-110, Nov. 2017.

[17] O. Abrishambaf, M. Ghazvini, L. Gomes, P. Faria, Z. Vale and J. Corchado, "Application of a Home Energy Management System for Incentive-Based Demand Response Program Implementation," 2016 27th International Workshop on Database and Expert Systems Applications (DEXA), pp. 153-157, 2016.

[18] Md T. Ahmed, P. Faria, O. Abrishambaf, and Z. Vale, "Electric Water Heater Modelling for Direct Load Control Demand Response," IEEE 16TH INTERNATIONAL CONFERENCE OF INDUSTRIAL

INFORMATICS (INDIN2018), 18-20 July, 2018, Porto, Portugal.

[19] M. Hu, and F. Xiao, "Investigation of the Demand Response Potentials of Residential Air Conditioners Using Grey-box Room Thermal Model," Energy Procedia., vol.105, pp. 2759-2765, May 2017.

[20] Mostafa S. Zolpirani, Design of a Direct Load Control Program for Residential Electric Water Heaters. PhD Thesis, Dept. of Electrical and Computer Engineering, University of New Brunswick, July 2016.

[21] C. Woo, L. Greening, "Guest editors' introduction”, Energy, 35 (4) (April 2010) Demand response resources: the US and International Experience.
[22] P. Faria and Z. Vale, "Demand response in electrical energy supply: An optimal real time pricing approach," Energy, vol. 36, no. 8, pp. 5374-5384, Aug. 2011.

[23] smartgrid.epri.com/UseCases/Direct\%20Load\%20Control\%20V3.2.pdf. [ONLINE] Available at: http://iecsa.net/en/home/. [Accessed 27 August 2018].

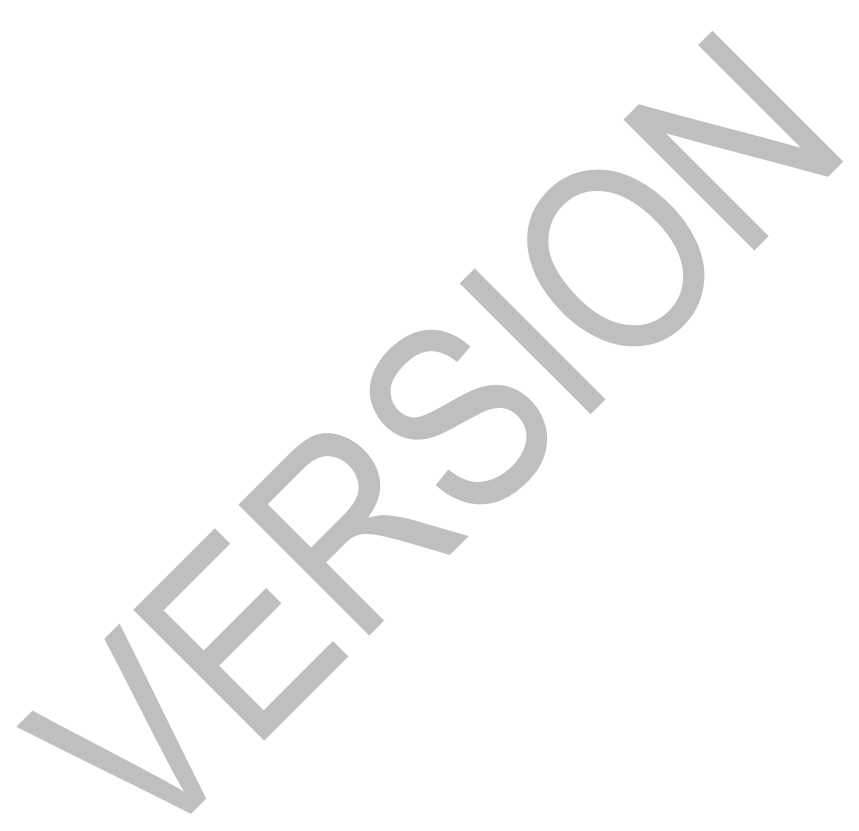

